\title{
Suppression of proliferation and oxidative stress by extracts of Ganoderma lucidum in the ovarian cancer cell line OVCAR-3
}

\author{
TZE-CHEN HSIEH and JOSEPH M. WU
}

\author{
Department of Biochemistry and Molecular Biology, New York Medical College, Valhalla, NY 10595, USA
}

Received June 30, 2011; Accepted August 3, 2011

DOI: 10.3892/ijmm.2011.788

\begin{abstract}
Lingzhi (LZ) is a medical mushroom also known as Ganoderma lucidum, which has been used in traditional Chinese medicine for more than 4,000 years and moreover, due to its presumed health benefits and apparent absence of side-effects it has also been widely consumed as a dietary supplement by cancer patients and by individuals diagnosed with various chronic diseases. The reported benefits of Ganoderma lucidum may be largely ascribed to its biologically active constituent polysaccharides and triterpenes known as ganoderic acids having structural similarity to steroid hormones. Laboratory studies have shown that Ganoderma lucidum enhances immune functions and also inhibits growth of various cancer cells both in vitro and in vivo. However, the mechanism by which Ganoderma lucidum exerts its chemopreventive activities remains unknown. In this study, we investigated whether Ganoderma lucidum elicits its anti-tumor effects by suppressing cell growth and inducing antioxidative/detoxification activity in human ovarian OVCAR-3 cells. The results showed that Ganoderma lucidum inhibits cell growth and disruption of cell cycle progression via down regulation of cyclin D1. Chemopreventive activities elicited by Ganoderma lucidum were demonstrated by the induction of antioxidant SOD and catalase as well as the phase II detoxification enzyme NAD(P)H:quinone oxidoreductase 1 (NQO1) and glutathione S-transferase P1 (GSTP1) via the Nrf2 mediated signaling pathway known to provide chemoprotection against carcinogenicity. These findings indicate that Ganoderma lucidum possesses chemopreventive potential contributing to its overall health effects and further suggest that Ganoderma lucidum may have clinical applications as an adjunct supplementary agent in chemotherapy.
\end{abstract}

\section{Introduction}

Ovarian cancer $(\mathrm{OvCa})$ is the most frequent cause of gynecologic cancer-related death in women $(1,2)$. Compared to other

Correspondence to: Dr Joseph M. Wu, Department of Biochemistry and Molecular Biology, New York Medical College, Basic Sciences Building, Room 147, Valhalla, NY 10595, USA

E-mail: joseph_wu@nymc.edu

Key words: Ganoderma lucidum, Lingzhi, OVCAR-3 cells, antiproliferative, anti-oxidative, phase II detoxification gynecologic malignancies, OvCa has the highest fatality-tocase ratio $(1,2)$. The steady incidence rate of $\mathrm{OvCa}$ and the continuing upward trend of OvCa-related fatalities are attributable to a combination of genetic, environment and lifestyle factors (3). Genetics are considered to play a relatively minor role, while environment and lifestyle factors contribute to a greater extent to ovarian carcinogenesis suggesting that a window of opportunity exists for chemoprevention of $\mathrm{OvCa}$. Among approaches for chemoprevention, the use of herbal products has gained popularity, particularly those prepared from medicinal mushrooms in light of the traditional high esteem they have held in Asian cultures (4-8) for promoting and maintaining overall health and wellness, notably through potentiation of immune functions and regulation of biological responses (6-11). Data have also shown that chemoprevention activities of medicinal mushroom extracts extend beyond the immune system; for instance, evidence abounds that they suppress tumorigenesis further supporting their diverse effects in treating diseases in humans (10,12-14).

Lingzhi (LZ) extracts are derived from edible mushroom known as Ganoderma lucidum $(15,16)$. The anticancer properties of Ganoderma lucidum have been attributed to its bioactive compounds including polysaccharides and a group of triterpenes (15-18). To date, the molecular details by which Ganoderma lucidum exerts its biological effects remain incompletely understood. The notion that Ganoderma lucidum may exert chemopreventive properties implies that it exerts a multitude of effects ranging from antioxidant, free-radical scavenging, and signal modulatory activities, as well as the ability to affect phase II detoxification enzymes $(19,20)$. The purpose of the present study was to investigate the effects of Ganoderma lucidum on cell growth inhibition in OVCAR-3 cells; to determine the cancer-protective potentials of Ganoderma lucidum on the changes of antioxidant enzymes (SOD and catalase) and phase II detoxification enzymes (NQO1 and GSTP1) and to explore the possible mechanism mediating its anti-oxidative and detoxification effects through the Nrf2/Keap1 pathway.

\section{Materials and methods}

Reagents. Bovine serum albumin (BSA), glutathione (GSH), 1-chloro-2,4-dinitrobenzene (CDNB), oxidized glutathione, menadione, dicoumarol, NADPH and glutathione reductase were purchased from Sigma Chemicals (St. Louis, MO, USA). The primary antibodies anti-Rb, anti-cyclin D1, anti-NQO1, 
anti-GSTP1, anti-SOD, anti-catalase, anti-Keap1, antiNrf2, anti-DJ1, anti-thioredoxin (Trx) and anti-actin, and the secondary antibodies were purchased from Santa Cruz Biotechnology (Santa Cruz, CA, USA). Fetal bovine serum (FBS), RPMI-1640 medium, penicillin and streptomycin were purchased from Cellgro Inc. (Herndon, VA, USA). All the other chemicals and solvents used were of analytical grade.

Cell culture. NIH:OVCAR-3 cells (HTB-161) were purchased from the American Type Culture Collection (Manassas, VA, USA) and maintained in RPMI-1640 media containing $2 \mathrm{mM}$ L-glutamine, supplemented with $20 \%$ heat inactivated fetal bovine serum (FBS), penicillin $(100 \mathrm{U} / \mathrm{ml})$ and streptomycin $(100 \mu \mathrm{g} / \mathrm{ml})$ in a humidified atmosphere of $5 \% \mathrm{CO}_{2}$ in air at $37^{\circ} \mathrm{C}(21)$. Cells were subcultured once every week by trypsinization and reseeding in new culture flasks.

Source of Ganoderma lucidum Lingzhi (LZ). LZ capsules used (lot 3BA03020528) were provided by Integrated Chinese Medicine Holdings Ltd. (Hong Kong, China), as a mushroom product produced from mycelia of Ganoderma lucidum according to Good Manufacturing Practice (GMP) standards. Additional quality control of LZ involved determination of heavy metal contents and microorganism contamination, performed by government approved testing centers in Hong Kong.

Preparation of aqueous and ethanolic extracts of LZ. To prepare aqueous or ethanolic extracts of LZ, the contents of each capsule was suspended in $6 \mathrm{ml}$ of water or $70 \%$ ethanol, followed by intermittent mixing and vortexing for $60 \mathrm{~min}$ at room temperature and a centrifugation step to remove insoluble particulates. The clear supernatant was sterilized by passing through a $0.22 \mu \mathrm{m}$ filter and stored in aliquots at $4^{\circ} \mathrm{C}$. Each aliquot was used only once and discarded. Before use, the stock was further diluted in tissue culture media to give the final indicated concentrations.

Effects of LZ on cell growth and colony formation. For the cell proliferation assay, OVCAR-3 cells were seeded at a density of $5 \times 10^{4}$ cells $/ \mathrm{ml}$ in T-25 flasks and allowed to attach overnight. Cells were incubated with 0,5 or $10 \mu \mathrm{l} / \mathrm{ml}$ water or ethanolic extracts of LZ for 1 and 3 days. Control and treated cells were harvested by trypsinization and cell numbers were determined using a hemocytometer (22-24). Harvested cells were washed twice with PBS, and pellets were stored at $-80^{\circ} \mathrm{C}$ for further analysis. The colony formation assay was performed as described previously with some modification (23). Cells (800-1200 cells $/ \mathrm{ml}, 2 \mathrm{ml} /$ well) were added to 6 -well tissue culture plates containing varying concentrations of water or ethanolic LZ extracts, followed by an additional 8 days of incubation to allow colonies to form. Colonies were fixed and stained with $1.25 \%$ crystal violet, followed by extensive washing to remove excessive dye, and imaged by an HP scanner. Quantitative changes in clonogenicity were determined by extraction of the colonies with $0.5 \mathrm{ml} 10 \%$ acetic acid and measuring the absorbance of the extracted dye at $595 \mathrm{~nm}$. The experiments were performed in triplicate (25).

Preparation of cell extracts and immunoblot analysis. Cells were collected by centrifugation and were lysed in ice-cold
RIPA buffer (50 mM Tris, pH 7.4, $150 \mathrm{mM} \mathrm{NaCl}, 1 \mathrm{mM}$ EDTA, $1 \%$ Triton X-100, $1 \%$ deoxycholate, $0.1 \%$ SDS, $1 \mathrm{mM}$ dithiothreitol and $10 \mu \mathrm{l} / \mathrm{ml}$ protease inhibitor cocktail). The extracts were centrifuged and the clear supernatants were stored in aliquots at $-80^{\circ} \mathrm{C}$ for further analysis. Protein content of cell lysates were determined by a Coomassie protein assay kit (Pierce, Rockford, IL, USA) with BSA as a standard.

The aliquots of lysates ( $20 \mu \mathrm{g}$ of protein) were boiled with sample buffer for $5 \mathrm{~min}$, and resolved by $10 \%$ SDS-PAGE. The proteins were transferred to a nitrocellulose membrane and blocked in TBST buffer (10 mM Tris, $\mathrm{pH} 7.5,100 \mathrm{mM} \mathrm{NaCl}$ and $0.05 \%$ Tween-20) containing $3 \%$ nonfat dried milk overnight at $4^{\circ} \mathrm{C}$. The blots were incubated with various primary antibodies, followed by incubation for $1 \mathrm{~h}$ with appropriate secondary antibodies conjugated to horseradish peroxidase in TBST. Actin expression was used as loading control. The intensity of the specific immunoreactive bands were detected by enhanced chemiluminescence (ECL), using the manufacturer's protocol (Kirkegared \& Perry Laboratories, Gaithersburg, MD, USA) quantified by densitometry and normalized against the actin loading control, as previously described (8).

Statistical analysis. The results are expressed as mean \pm standard deviation (SD). The significance between the control and treated groups was performed by the Student's t-test and P-values $<0.001$ were taken as significant in all the cell growth and colony formation experiments.

\section{Results}

Effects of aqueous and ethanolic extracts of LZ on OVCAR-3 cell growth and colony formation. The ability of LZ extracts to inhibit OVCAR-3 cell proliferation was investigated. Neither aqueous nor ethanolic extracts significantly inhibited cell proliferation after 1 day of treatment, whereas exposure of cells for 3 days resulted in a pronounced suppression of cell growth (Fig. 1A). More significant dose-dependent growth suppression was observed with the ethanolic extract, while the aqueous extract had less potent inhibitory activity. Typically, at a concentration of $10 \mu \mathrm{l} / \mathrm{ml}$ ethanolic extract, approximately $50 \%$ inhibition of cell growth was found.

Additional evidence for the growth inhibitory activity of aqueous and ethanolic extracts of LZ on human OVCAR-3 cells was tested by the colony formation assay. The ability of OVCAR-3 cells to form colonies was more significantly inhibited by ethanolic extracts than aqueous extracts of LZ, as determined by a marked reduction in both the number and the size of colonies (Fig. 1B).

Effects of aqueous and ethanolic extracts of LZ on cell cycle regulatory proteins $R b$ and cyclin $D 1$ expression in OVCAR-3 cells. To obtain additional information on the antiproliferative effects of LZ, changes in selective cell cycle regulatory protein expression were investigated in control and LZ-treated cells, by Western blot analysis. Systematic probing of several proteins known to play an important role in cell cycle control did not show changes following 1 or 3 days of treatment with aqueous and ethanolic extracts of LZ. A noted exception was found in cyclin D1 whose expression was reduced by $\sim 20$ and $\sim 38 \%$, respectively, on Day 1, and by $\sim 28$ and $\sim 24 \%$, respectively, 


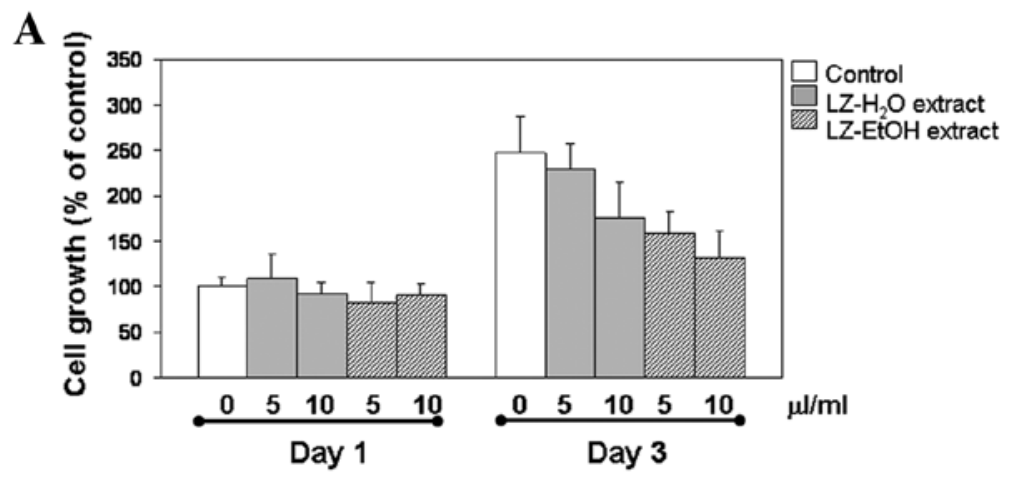

B
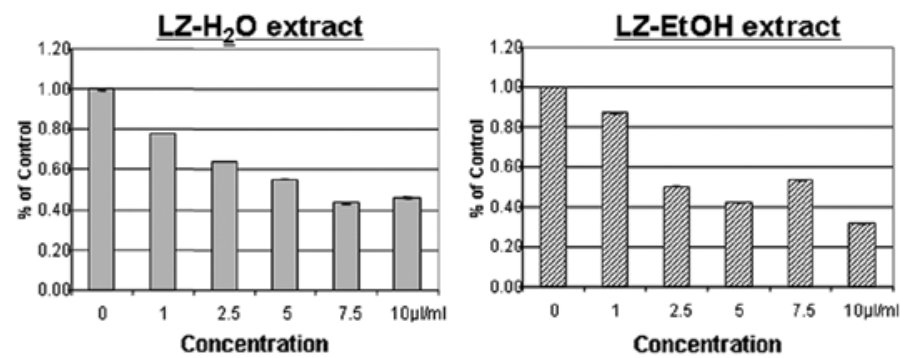

Figure 1. Effects of aqueous and ethanolic extracts of LZ on OVCAR-3 cell growth and colony formation. (A) Cells were treated with various concentrations of aqueous and ethanolic extracts of LZ (0,5 and $10 \mu \mathrm{l} / \mathrm{ml})$. Cell numbers were determined on Days 1 and 3 with a hemocytometer. (B) Clonogenicity following LZ treatment was measured as described in Materials and methods. Quantitative analysis of colony formation as measured by absorbance of acetic acid extracted crystal violet dye retained by the cells at $595 \mathrm{~nm}$. Results show the mean $\pm \mathrm{SD}$ of at least 3 separate experiments.

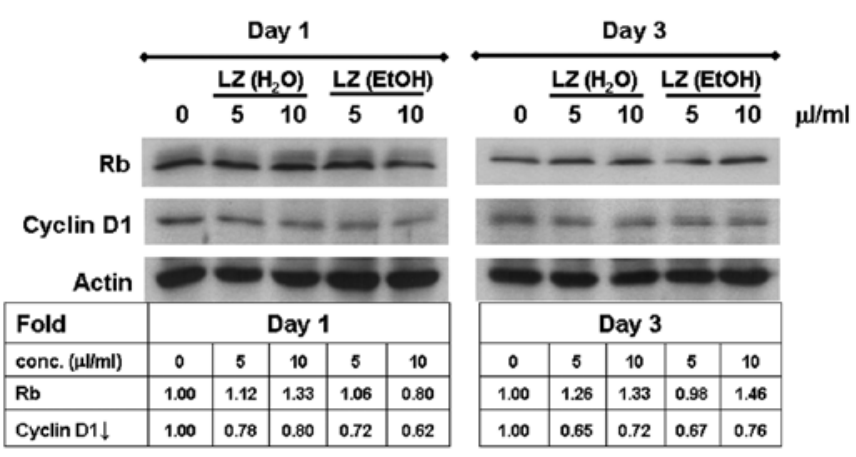

Figure 2. Effects of aqueous and ethanolic extracts of LZ on cell cycle regulatory proteins $\mathrm{Rb}$ and cyclin D1 expression in OVCAR-3 cells. Cells were treated with 0,5 or $10 \mu \mathrm{l} / \mathrm{ml}$ of aqueous and ethanolic extracts of LZ for 1 and 3 days and cells were harvested and total protein extracts were prepared as described in Materials and methods. Aliquots of total extracts were separated by SDS-PAGE and the effects on $\mathrm{Rb}$ and cyclin $\mathrm{D} 1$ protein expression were determined by Western blot analysis. Actin was used as a loading control to determined changes in the expression by treatment. The intensity of the specific immunoreactive bands were quantified by densitometry and expressed as a fold-difference against actin.

on Day 3 after treatment with $10 \mu \mathrm{l} / \mathrm{ml}$ aqueous and ethanolic extracts of LZ. As cyclin D1 changes may impinge on the expression and also the state of phosphorylation of retinoblastoma $(\mathrm{Rb})$, which is considered an established indicator of the proliferative state of the cell by controlling the availability of transcription factor E2F, we also analyzed hypo- and hyperphosphorylated Rb by Western blot analysis. Treatment by LZ did not affect either form of Rb (Fig. 2).

Effects of aqueous and ethanolic extracts of LZ on the expression of antioxidant enzymes and phase II detoxifica-
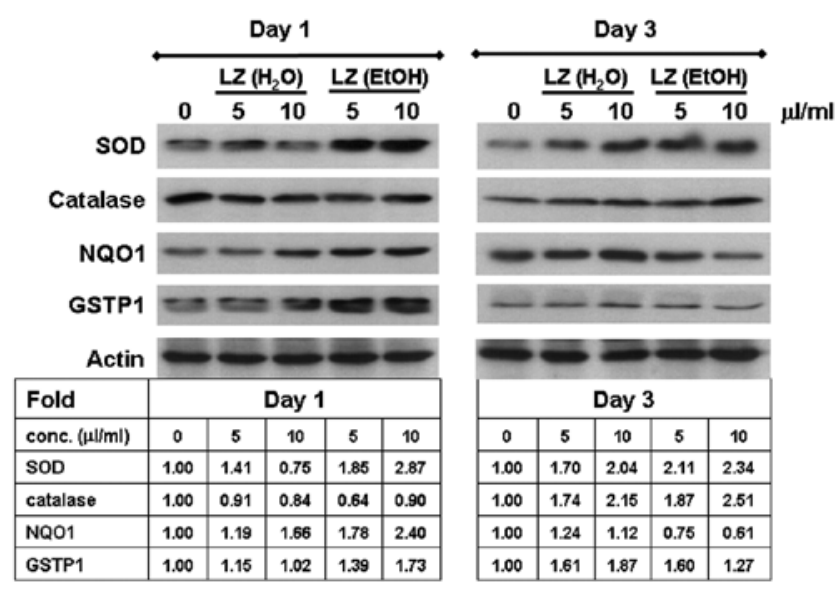

Figure 3. Effects of aqueous and ethanolic extracts of $\mathrm{LZ}$ on the expression of antioxidant enzymes and phase II detoxification enzymes. Following a 1- or 3-day treatment with different doses 0,5 or $10 \mu \mathrm{l} / \mathrm{ml} \mathrm{LZ}$ extracts, cells were harvested and the expression of SOD, catalase, NQO1 and GSTP1 were determined by immunoblot analysis. Blots were quantified and relative expression levels are shown as a fold-difference against the actin loading control.

tion enzymes. The effects of LZ treatment on the intracellular redox status were measured by assessing the levels of the antioxidant enzymes, SOD and catalase (Fig. 3). Treatment of the OVCAR-3 cells with $10 \mu \mathrm{l} / \mathrm{ml}$ ethanolic extracts of $\mathrm{LZ}$ revealed changes in the expression of SOD. The densitometric analysis of the blots showed a significant increase of 2.87and 2.34-fold in cells treated for 1 and 3 days, respectively (Fig. 3). The effects of LZ on catalase expression were also investigated. Treatment with $10 \mu \mathrm{l} / \mathrm{ml}$ aqueous and ethanolic LZ extracts for 3 days resulted in a significant increase in catalase expression by 2.15 - and 2.51-fold, respectively whereas 


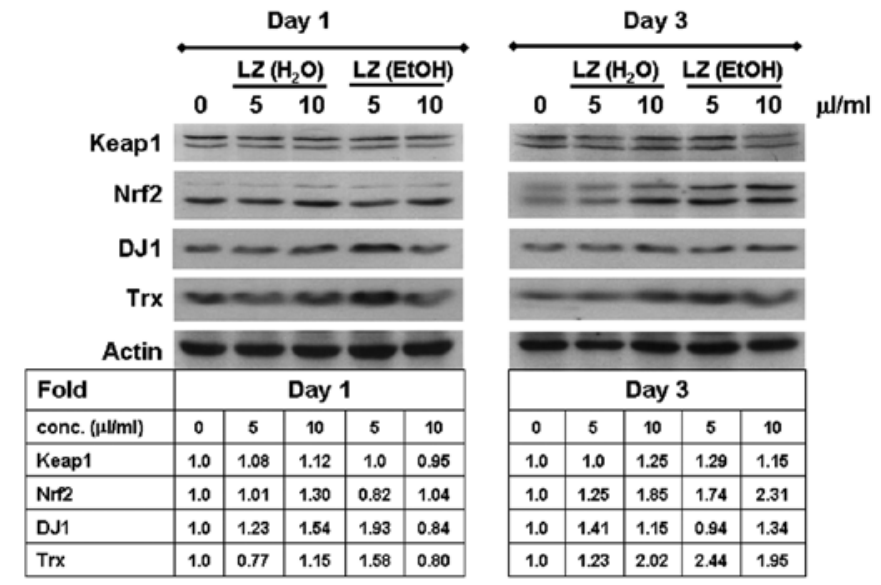

Figure 4. Effects of aqueous and ethanolic extracts of LZ on the regulation of antioxidants and phase II detoxification enzymes via Nrf2/Keap1 signaling. Following a 1- or 3-day treatment with different doses 0,5 or $10 \mu 1 / \mathrm{ml} \mathrm{LZ}$ extracts, cells were harvested and total protein extracts were prepared as described in Materials and methods. Aliquots of total extracts were separated by SDS-PAGE and analyzed for Keap1, Nrf2, DJ1 and Trx by immunoblot analysis. Blots were quantified and relative expression levels are shown as a fold-difference against the actin loading control.

treatment for 1 day with aqueous and ethanolic extracts of LZ slightly decreased the catalase expression (Fig. 3). These results suggest that $\mathrm{LZ}$ increased the cellular defenses by activating the antioxidant enzymes, SOD and catalase. We also determined the effects of LZ treatment on the phase II detoxification enzymes, NQO1 and GSTP1. The level of expression of NQO1 was up-regulated by 1.66- and 2.4-fold, respectively after 1 day of treatment with $10 \mu \mathrm{l} / \mathrm{ml}$ aqueous and ethanolic extracts of LZ, but $10 \mu \mathrm{l} / \mathrm{ml}$ ethanolic extracts of LZ resulted in the reduction on NQO1 expression by $39 \%$ at Day 3 (Fig. 3). The changes on the expression of GSTP1 by LZ were also investigated. Up-regulation of GSTP1 was observed after treatment with the aqueous and ethanolic extracts of LZ; the former, however, was much less potent for inducing GSTP1 than the latter at Day 1 whereas by Day 3 there was no significant difference on the induction of GSTP1 between the aqueous and ethanolic extracts of LZ-treated cells (Fig. 3). Taken together, these results suggest that LZ could also increase the cellular defenses by activating the phase II detoxification enzymes, NQO1 and GSTP1.

Effects of aqueous and ethanolic extracts of $\mathrm{LZ}$ on the regulation of antioxidants and phase II detoxification enzymes via Nrf2/Keapl signaling. The induction of antioxidants and phase II detoxification enzymes is under the control of $\mathrm{Nrf2/}$ Keap1. Therefore, we tested whether treatment by LZ elicits changes in the levels of Nrf2/Keap1 in OVCAR-3 cells. LZ treatment significantly enhanced the level of Nrf2 in a doseand time-dependent manner; the most pronounced induction occurred in cells exposed to $10 \mu \mathrm{l} / \mathrm{ml} 70 \%$ ethanol $\mathrm{LZ}$ for $72 \mathrm{~h}$, while no change in Nrf2 expression was observed under any treatment condition at $24 \mathrm{~h}$ (Fig. 4). Analysis also revealed that whereas LZ did not affect the level of Keap1 using the identical treatment conditions, it increased the expression of DJ1 and Trx, both thought to play a role in the stabilization and functional commitment of Nrf2. These results suggest that LZ may stimulate Nrf2-mediated antioxidant response element (ARE) activation by enhancing nuclear Nrf2 protein level and its stability as well as competence for engagement with ARE-responsive genes.

\section{Discussion}

The chemical and biological constituents of Ganoderma lucidum, Lingzhi, have been studied extensively over the past decades. Polysaccharide fractions and some 20 triterpenes isolated from Ganoderma lucidum were shown to be cytotoxic against a panel of human and murine tumor cell lines (18). Polysaccharides are typically found in the water extract, which has been reported to exert immunostimulating, anti-inflammatory and anti-tumor properties $(18,26)$. On the other hand, components of the ethanolic extract include essential oils, terpenoids and glycosides, with the greatest interest focused on terpinoids known to display potent biological activities $(18,26)$. To our knowledge, whether the aqueous and ethanolic extracts of LZ demonstrate effective anti-proliferative/antioxidative stress properties has not been examined. Results in this manuscript provide evidence that the ethanolic extract of LZ has more potent activity than the aqueous extract on suppressing cell proliferation and on inducing the expression of enzymes instrumental in counteracting oxidative stress in OVCAR-3 cells.

Numerous plants have been shown to contain high levels of natural antioxidants capable of scavenging free radicals (19). This property, in effect, may well decrease the rate of oxidative stress induced DNA damage and thereby partially account for chemopreventive mechanisms attributed to plant-derived antioxidants. Both triterpene and polysaccharide extracts have shown antioxidative effect against pyrogallol-induced erythrocyte membrane oxidation and $\mathrm{Fe}(\mathrm{II})$-ascorbic acid-induced lipid peroxidation $(19,27)$. The results of this study clearly demonstrated that both aqueous and ethanolic extracts exhibited antioxidative activities by increasing SOD and catalase expression in a dose-dependent manner, with the ethanolic extracts showing more significant effect. One might therefore surmise that $\mathrm{LZ}$ extracts display the capacity for scavenging free radicals per se or disrupt propagation of $\mathrm{H}_{2} \mathrm{O}_{2}$ decomposition products to effectively prevent $\mathrm{H}_{2} \mathrm{O}_{2}$-induced oxidative damage to cellular DNA. Such a mechanism may lend support to the notion that induction of antioxidant activity by LZ serves as a basis for its involvement in chemoprevention.

Phase II-detoxification enzymes play an important role in the removal of compounds with genotoxic potentials, thereby reducing the risk of somatic mutations and tumor transformation. In this study, we investigated whether the chemopreventive potential induced by LZ in OVCAR-3 cells may involve phase II enzymes. We found that both aqueous and ethanolic extracts increased NQO1 and GSTP1 expression in a dose-dependent manner and the ethanolic extracts were more potent than the aqueous extracts. These findings suggest a novel mechanism through which $\mathrm{LZ}$ may contribute to the cancer chemoprevention against carcinogenicity, mutagenicity and other toxicities caused by carcinogenic xenobiotics.

In conclusion, this study demonstrated that $\mathrm{LZ}$ has anti-cancer activities beyond that of the immune system with the induction of chemopreventive activity and suppres- 
sion of tumorigenesis via the induction of antioxidants and phase II-detoxification enzymes. Nrf2-mediated activation of cytoprotective genes contributes to the beneficial effects of LZ to prevent or fight various cancers including ovarian cancer.

\section{Acknowledgements}

We are grateful for the support of an unrestricted grant from the Hong Kong Healthcare Center Ltd. We especially acknowledge the vision and commitment of Ms. Vivien Chou and her family for mechanistic studies of Chinese herbals in the prevention and management of chronic diseases including ovarian cancer.

\section{References}

1. Holschneider $\mathrm{CH}$ and Berek JS: Ovarian cancer: epidemiology, biology, and prognostic factors. Semin Surg Oncol 19: 3-10, 2000

2. La Vecchia C: Epidemiology of ovarian cancer: a summary review. Eur J Cancer Prev 10: 125-129, 2001.

3. Parkin DM, Bray F, Ferlay J and Pisani P: Global cancer statistics, 2002. CA Cancer J Clin 55: 74-108, 2005.

4. Yang MM, Chen Z and Kwok JS: The anti-tumor effect of a small polypeptide from Coriolus versicolor (SPCV). Am J Chin Med 20: 221-232, 1992 .

5. Wasser SP and Weis AL: Therapeutic effects of substances occurring in higher Basidiomycetes mushrooms: a modern perspective. Crit Rev Immunol 19: 65-96, 1999.

6. Borchers AT, Stern JS, Hackman RM, Keen CL and Gershwin ME: Mushrooms, tumors, and immunity. Proc Soc Exp Biol Med 221: 281-293, 1999

7. Kidd PM: The use of mushroom glucans and proteoglycans in cancer treatment. Altern Med Rev 5: 4-27, 2000.

8. Hsieh TC, Wu P, Park S and Wu JM: Induction of cell cycle changes and modulation of apoptogenic/anti-apoptotic and extracellular signaling regulatory protein expression by water extracts of I'm-Yunity (PSP). BMC Complement Altern Med 6: 30, 2006

9. Chu KK, Ho SS and Chow AH: Coriolus versicolor: a medicinal mushroom with promising immunotherapeutic values. J Clin Pharmacol 42: 976-984, 2002.

10. Yue GG, Fung KP, Leung PC and Lau CB: Comparative studies on the immunomodulatory and antitumor activities of the different parts of fruiting body of Ganoderma lucidum and Ganoderma spores. Phytother Res 22: 1282-1291, 2008.

11. Lin ZB: Cellular and molecular mechanisms of immuno-modulation by Ganoderma lucidum. J Pharmacol Sci 99: 144-153, 2005.

12. Chang R: Bioactive polysaccharides from traditional Chinese medicine herbs as anticancer adjuvants. J Altern Complement Med 8: 559-565, 2002.

13. Chang R and White JD: Asian therapies for cancer - coming of age. J Altern Complement Med 8: 541-543, 2002.
14. Zaidman BZ, Yassin M, Mahajna J and Wasser SP: Medicinal mushroom modulators of molecular targets as cancer therapeutics. Appl Microbiol Biotechnol 67: 453-468, 2005.

15. Chen HS, Tsai YF, Lin S, Lin CC, Khoo KH, Lin CH and Wong CH: Studies on the immuno-modulating and anti-tumor activities of Ganoderma lucidum (Reishi) polysaccharides. Bioorg Med Chem 12: 5595-5601, 2004.

16. Sliva D: Cellular and physiological effects of Ganoderma lucidum (Reishi). Mini Rev Med Chem 4: 873-879, 2004.

17. Cheng KC, Huang HC, Chen JH, Hsu JW, Cheng HC, Ou CH, Yang WB, Chen ST, Wong CH and Juan HF: Ganoderma lucidum polysaccharides in human monocytic leukemia cells: from gene expression to network construction. BMC Genomics 8: 411, 2007.

18. Jiang J, Slivova V and Sliva D: Ganoderma lucidum inhibits proliferation of human breast cancer cells by down-regulation of estrogen receptor and NF- $\kappa \mathrm{B}$ signaling. Int J Oncol 29: 695-703, 2006.

19. Liu F, Ooi VE and Chang ST: Free radical scavenging activities of mushroom polysaccharide extracts. Life Sci 60: 763-771, 1997.

20. Lakshmi B, Ajith TA, Jose N and Janardhanan KK: Antimutagenic activity of methanolic extract of Ganoderma lucidum and its effect on hepatic damage caused by benzo[a]pyrene. J Ethnopharmacol 107: 297-303, 2006.

21. Hamilton TC, Young RC, McKoy WM, Grotzinger KR, Green JA, Chu EW, Whang-Peng J, Rogan AM, Green WR and Ozols RF: Characterization of a human ovarian carcinoma cell line (NIH:OVCAR-3) with androgen and estrogen receptors. Cancer Res 43: 5379-5389, 1983.

22. Hsieh TC, Burfeind P, Laud K, Backer JM, Traganos F, Darzynkiewicz Z and Wu JM: Cell cycle effects and control of gene expression by resveratrol in human breast carcinoma cell lines with different metastatic potentials. Int J Oncol 15: 245-252, 1999.

23. Hsieh TC, Wijeratne EK, Liang JY, Gunatilaka AL and Wu JM: Differential control of growth, cell cycle progression, and expression of NF-kappaB in human breast cancer cells MCF-7, MCF-10A, and MDA-MB-231 by ponicidin and oridonin, diterpenoids from the chinese herb Rabdosia rubescens. Biochem Biophys Res Commun 337: 224-231, 2005.

24. Hsieh TC and Wu JM: Differential control of growth, cell cycle progression, and gene expression in human estrogen receptor positive MCF-7 breast cancer cells by extracts derived from polysaccharopeptide I'm-Yunity and Danshen and their combination. Int J Oncol 29: 1215-1222, 2006.

25. Niles RM, McFarland M, Weimer MB, Redkar A, Fu YM and Meadows GG: Resveratrol is a potent inducer of apoptosis in human melanoma cells. Cancer Lett 190: 157-163, 2003.

26. Shiao MS: Natural products of the medicinal fungus Ganoderma lucidum: occurrence, biological activities, and pharmacological functions. Chem Rec 3: 172-180, 2003.

27. Zhu M, Chang Q, Wong LK, Chong FS and Li RC: Triterpene antioxidants from Ganoderma lucidum. Phytother Res 13: 529-531, 1999. 